\title{
Patrones de presencia y abundancia de aves terrestres en la isla Saliaca, Sinaloa, México
}

\author{
Patterns of occurrence and abundance of land birds on Saliaca Island, Sinaloa, Mexico
}

\author{
José Alfredo Castillo-Guerrero $^{1 *}$, Erick González-Medina ${ }^{1}$ y Marco Antonio González-Bernal ${ }^{2}$ \\ ${ }^{1}$ Centro de Investigación Cientifica y de Educación Superior de Ensenada, B.C. Apartado postal 2732, Ensenada, B.C., México. \\ ${ }^{2}$ Escuela de Biología. Universidad Autónoma de Sinaloa, Ciudad Universitaria. Culiacán, Sinaloa. México. \\ *Correspondencia: acastill@cicese.mx
}

\begin{abstract}
Resumen. Se determinó la abundancia y composición taxonómica de las aves terrestres en la Isla Saliaca, Sinaloa, México, mediante puntos de conteo en 3 periodos de muestreo (verano, otoño e invierno) y 3 tipos de hábitat (bosque espinoso, manglar y duna). Se observaron 82 especies, un número más alto que el registrado para cualquier otra isla del golfo de California. La riqueza y abundancia fueron mayores en otoño (59 especies, $9.2 \pm 1.2$ aves por punto) e invierno (48 y 7.5 \pm 0.9 ) que en verano (33 y $5.2 \pm 0.3$ ); este patrón refleja el arribo de aves migrantes neotropicales. En cuanto a los tipos de hábitat, la mayor riqueza y abundancia se encontró en el bosque espinoso (60 especies y $9.3 \pm 0.9$ aves por punto), seguida por manglar (16 y $3.3 \pm 0.8)$ y duna (3 y $1.0 \pm 0.2)$, lo cual es acorde con la estructura vegetal más compleja y la área de cobertura mayor del bosque espinoso. La isla Saliaca puede representar un relicto del hábitat costero regional, por lo que es importante su protección y conservación.
\end{abstract}

Palabras clave: aves terrestres, golfo de California, islas, Sinaloa.

\begin{abstract}
We determined the abundance and taxonomic composition of land-birds in Saliaca Island, Sinaloa, Mexico, by means of point counts during 3 sampling periods (summer, autumn and winter) and 3 types of habitat (thorn forest, mangrove and dune). We observed 82 species, which is greater than the number reported for any other Island of the Gulf of California. The species richness and abundance were greater in autumn (59 species and $9.2 \pm 1.2$ birds per point count) and winter (48 and $7.5 \pm 0.9$ ) than in summer (33 and $5.2 \pm 0.3$ ), a pattern resulting from the arrival of neotropical migrants. With regard to differences by habitat, the greatest richness and abundance occurred in the thorn forest (60 species and 9.3 \pm 0.9 birds per point), followed by mangrove (16 and $3.3 \pm 0.8$ ) and dune ( 3 and $1.0 \pm 0.2$ ), which is agreement with the higher complexity of vegetation structure and the greater area of thorn forest. Saliaca Island could represent a relict of the coastal regional habitat, which enhances its conservation and protection value.
\end{abstract}

Key words: Land-birds, Gulf of California, Islands, Sinaloa.

\section{Introducción}

Históricamente, la avifauna del golfo de California ha llamado la atención de los ornitólogos. Al inicio del siglo XX se realizaron varias expediciones para conocer la avifauna de la península de Baja California, incluyendo visitas a algunas islas del golfo (Brewster,1902; Grinnell, 1928). De hecho, la importancia del golfo de California para las aves acuáticas está bien documentada (Anderson, 1983; Massey y Palacios, 1994), a diferencia de lo que ocurre con aves terrestres, donde el tiempo y la intensidad de los muestreos dedicados a documentar los patrones de presencia y abundancia en las islas han sido muy limitados (Cody y Velarde, 2002).

Recibido: 17 julio 2007; aceptado: 03 octubre 2008
En una revisión reciente, Cody y Velarde (2002) mencionan que las aves terrestres de las islas del Golfo son poco conocidas y que los listados disponibles son incompletos. Específicamente para la porción sur, los estudios que han documentando los patrones de presencia y abundancia de aves en las islas han sido en las cercanas a la península de Baja California, como Cerralvo (Banks, 1963), Carmen (Gaviño et al., 1984; Cody y Velarde, 2002) y Espíritu Santo (Carmona et al., 2005), por lo que la carencia de información es aun más acentuado para las islas cercanas a la costa de Sinaloa.

En el noroeste de México, durante las últimas cinco décadas la población ha crecido en más del 200\%; como resultado, la construcción de caminos ha aumentado considerablemente, facilitando el acceso a lugares que antes resultaban remotos y se ha presentado una sobre 
explotación de algunos stocks pesqueros. Es decir, el acceso y atractivo de las islas para pescadores comerciales, turistas y otros visitantes se ha incrementado, y en consecuencia también el número de gente que utiliza las islas y los problemas asociados con el uso por humanos (Velarde y Anderson, 1994; Tershy et al., 1997).

Pese a que las islas del golfo de California se consideran áreas naturales protegidas desde 1978 (DOF, 1978), no hay información detallada sobre la composición temporal y espacial de sus recursos bióticos que permita, entre otras cosas, identificar los sitios más importantes para la conservación. El presente trabajo describe los patrones de abundancia y presencia de aves terrestres en los diferentes hábitats de la isla Saliaca en las épocas de verano, otoño e invierno, como una contribución a ese conocimiento.

\section{Materiales y métodos}

La isla Saliaca se ubica al sureste del golfo de California, entre los $25^{\circ} 08^{\prime} 34.3^{\prime \prime}-25^{\circ} 12^{\prime} 41.5^{\prime \prime} \mathrm{N}$ y los $108^{\circ} 22^{\prime} 11.7^{\prime \prime}$ - $108^{\circ} 16^{\prime} 10.3^{\prime \prime O}$ (Fig. 1), es una de las islas más grandes de la bahía de Santa María-La Reforma con un área aproximada de $22 \mathrm{~km}^{2}$. La bahía forma parte de la provincia fisiográfica del cinturón costero de Sinaloa y se ubica dentro de una llanura deltáica que en la parte norte está formada por el proceso de sedimentación terrígena de los ríos Sinaloa y Mocorito. Este proceso ocasionó la formación de islas e islotes en la parte norte, incluida la isla Saliaca. La bahía se comunica con el golfo de California por medio de 2 bocas, una al noroeste, entre la isla Saliaca y la isla Altamura y otra al sureste, en el extremo sur de la isla Altamura (De la Lanza y Cáceres, 1994). La profundidad promedio en la bahía es de 3.5 $\mathrm{m}$, con profundidades máximas de $23 \mathrm{~m}$ en la boca de Perihuete, entre las islas Altamura y Saliaca (Serrano y Ramírez-Félix, 2003).

La isla Saliaca tiene aproximadamente 11 kilómetros de longitud en su eje mayor, la separan de la costa 2 canales poco profundos de menos de un kilómetro de ancho; tiene principalmente 3 tipos de vegetación: bosque espinoso, manglar y duna costera (Fig. 1). El bosque espinoso es el predominante e incluye más de 20 especies, siendo las más características la cacaragua (Vallesia glabra), el san Juanico (Jacquinia pungens), el sangregado (Jatropha curcas) y la cacachila (Karwinskia humboldtiana). El manglar ocupa también grandes extensiones de la isla, especialmente en el borde este e incluye sólo 4 especies: mangle negro (Avicenia germinans), mangle botoncillo (Conocarpus erecta), mangle cenizo (Laguncularia racemosa) y mangle rojo (Rhizophora mangle). La duna costera se encuentra en una pequeña franja por todo el borde oeste de la isla y

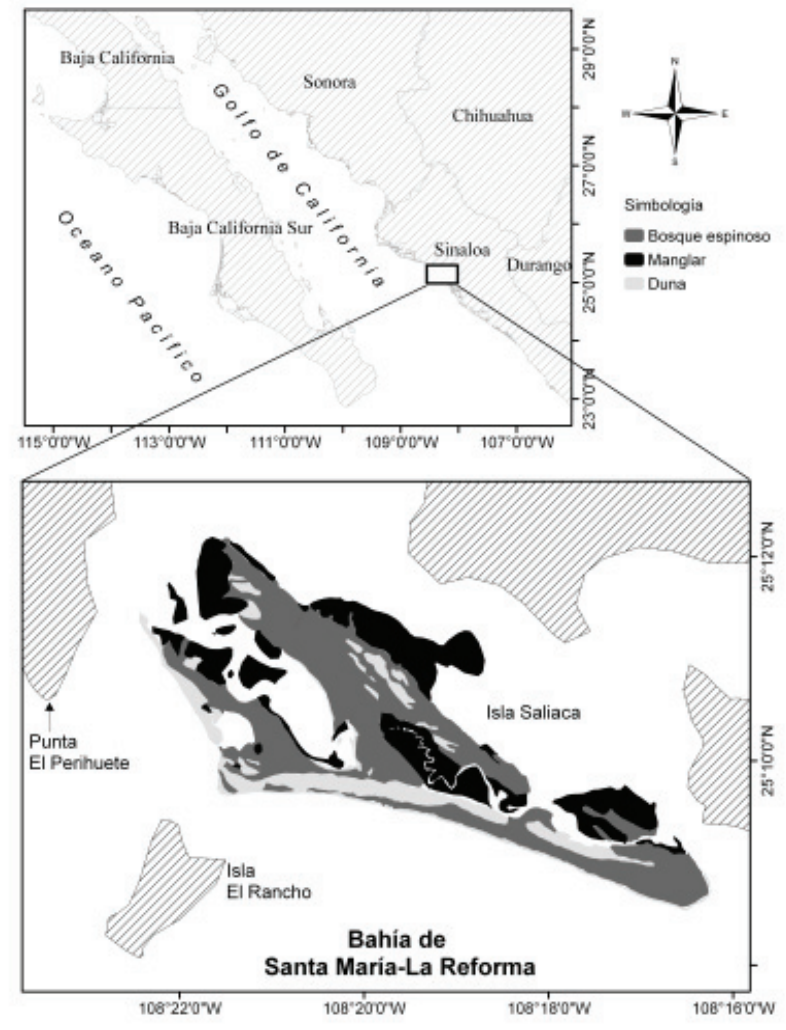

Figura 1. Isla Saliaca. Cobertura de cada tipo de hábitat en la isla.

en la parte central formando un cinturón que la recorre de manera longitudinal e incluye especies como la verdolaga de dunas (Abronia maritima), el chamizo (Atriplex barclayana), el vidrillo (Batis maritima), el haba de mar (Canavalia maritima), el zacate salado (Distichlis spicata) y el zacate vidrillo (Monanthchloe littoralis).

Para el registro de aves se realizaron 3 muestreos tratando de abarcar todos los tipos de hábitat en la isla; el primer muestreo se realizó en el verano (9-11 de junio, 2006), para determinar las especies de reproducción local; el segundo en el otoño, para conocer las especies de aves migratorias que utilizan la zona (26-28 de septiembre, 2006), y el tercero en invierno, para reforzar el monitoreo de las aves migratorias y ver cuáles permanecían en la zona (1-3 de febrero, 2007).

Para determinar la abundancia de aves se utilizó el método de puntos de conteo sin repetición en muestreos sucesivos, de manera que cada punto representó una unidad de muestreo independiente (Ralph et al., 2000). Los puntos tuvieron $15 \mathrm{~m}$ de radio y una duración de 5 minutos, con una distancia de $200 \mathrm{~m}$ entre ellos.

Los muestreos se realizaron a pie de las 06:00 a las 09:00 horas, eligiéndose un radio corto y duración larga para maximizar la detección de las aves; en otros estudios 
se ha determinado que el área de detección efectiva es alrededor de 25 m (Mikol, 1980; Casagrande y Beissinger, 1997), y con duración de 2-3 minutos se realiza cerca del $80 \%$ de los registros en diferentes tipos de hábitat (Shiu y Lee, 2003). De manera que con un radio menor y un tiempo ligeramente mayor se estima una detectabilidad completa y se usaron directamente, los datos de los puntos como índice de abundancia.

Los tipos de vegetación presentes en el área se clasificaron de acuerdo con Rzedowski (1978). Las especies se identificaron con el auxilio de binoculares y guías para las aves de México y Norteamérica (Howell y Webb, 1995; Sibley, 2001). La nomenclatura y secuencia taxonómica de las especies se realizó siguiendo el arreglo de American Ornithologists' Union (AOU, 1998).

Para cada estación se hizo una curva de acumulación de especies considerando un remuestreo con 1000 repeticiones con el programa EstimateS Versión 8.0 (Colwell, 2006). Posteriormente se ajustó una curva tipo Clench usando el programa Statistica 7.1 (StatSoft, 2005) y se realizó la predicción de la riqueza en función a la razón entre los parámetros $a$ y $b$ de la ecuación de la curva (JiménezValverde y Hortal, 2003).

Para comparar la abundancia se utilizó un análisis de varianza de 2 vías, con estación y hábitat como variables independientes, y la abundancia en cada punto como variable dependiente. El análisis se realizó considerando un $\alpha=0.05$.

\section{Resultados}

Riqueza de especies. Se registraron 82 especies de aves terrestres en los 3 periodos combinados, 70 durante los puntos de conteo y 12 más fuera de los conteos. La riqueza predicha mediante la curva tipo Clench fue similar a la que se presentó para el verano y el otoño; sin embargo, durante el invierno el esfuerzo de muestreo fue menor y afectó la certidumbre de nuestros datos, y la riqueza fue menor respecto a lo proyectado (Cuadro 1). Pese a lo anterior, el total estimado de riqueza coincide ampliamente con lo observado y el patrón fue muy claro, la riqueza es menor en verano respecto al otoño e invierno (Cuadro 1).

El tipo de vegetación con mayor riqueza fue el bosque espinoso (60), seguido de los ecotonos de bosque espinosomanglar (32), bosque espinoso-duna (26), manglar (16) y duna (3) (Cuadro 1). El patrón es consistente con la predicción realizada; sin embargo, se observaron menos especies de las proyectadas en todos los hábitats (Cuadro 1). Es decir, las diferencias entre hábitat son claras aunque el esfuerzo de muestreo fue insuficiente para detectar la totalidad de especies en cada hábitat.

Patrones de abundancia. Hubo diferencias significativas en la abundancia entre estaciones $\left(\mathrm{F}_{2,152}=3.73, \mathrm{P}=0.02\right)$. Se registró mayor cantidad de aves durante la visita de otoño $(9.2 \pm 1.2)$, seguida por invierno $(7.5 \pm 0.9)$ y verano (5.2 $\pm 0.3 ;$ Fig. 2A). Entre tipo de vegetación también hubo diferencias $\left(\mathrm{F}_{4,152}=3.87, \mathrm{P}=0.005\right)$, la mayor abundancia se presentó en el bosque espinoso $(9.3 \pm 0.9)$, seguida por manglar $(3.3 \pm 0.8)$ y duna $(1.0 \pm 0.2$, Cuadro 2 ; Fig. 2B).

Se advierten 3 patrones generales en la abundancia de especies: 1) de reproducción local muy abundantes en el verano que disminuyeron significativamente en el otoño e invierno, como Auriparus flaviceps, Cynanthus latirostris y Passerina versicolor; 2) de reproducción local con abundancia similar a lo largo del año, como Cardinalis

Cuadro 1. Parámetros de la estimación de riqueza de especies mediante la curva tipo Clench (Riqueza de especies $=($ a) $*$ Núm. de puntos/1+(b)*Núm. de puntos). Se muestra el intervalo de predicción al $95 \%$ y la riqueza observada en las diferentes estaciones y hábitats en isla Saliaca, Sinaloa $(\mathrm{BE}=$ Bosque espinoso)

\begin{tabular}{|c|c|c|c|c|c|c|}
\hline Estación & $R^{2}$ & $a$ & $b$ & $n$ & $\begin{array}{c}\text { Riqueza predicha } \\
(a / b)\end{array}$ & $\begin{array}{l}\text { Riqueza } \\
\text { observada }\end{array}$ \\
\hline Verano & 0.98 & 2.531 & 0.076 & 76 & $32.47-33.5$ & 33 \\
\hline Otoño & 0.99 & 4.025 & 0.075 & 73 & $53.04-54.03$ & 59 \\
\hline Invierno & 0.99 & 3.230 & 0.046 & 40 & $68.83-71.77$ & 48 \\
\hline \multicolumn{7}{|l|}{ Hábitat } \\
\hline $\mathrm{BE}$ & 0.99 & 3.404 & 0.048 & 93 & $69.7-71.7$ & 60 \\
\hline BE-Mangle & 0.97 & 3.633 & 0.079 & 28 & $44.3-46.9$ & 32 \\
\hline BE-Duna & 0.97 & 3.298 & 0.084 & 22 & $38.0-40.4$ & 26 \\
\hline Mangle & 0.99 & 2.793 & 0.082 & 11 & $33.2-34.4$ & 16 \\
\hline Duna & 0.99 & 0.478 & 0.047 & 9 & $9.45-10.9$ & 3 \\
\hline Total & 0.99 & 2.408 & 0.030 & 189 & $79.57-80.94$ & 82 \\
\hline
\end{tabular}




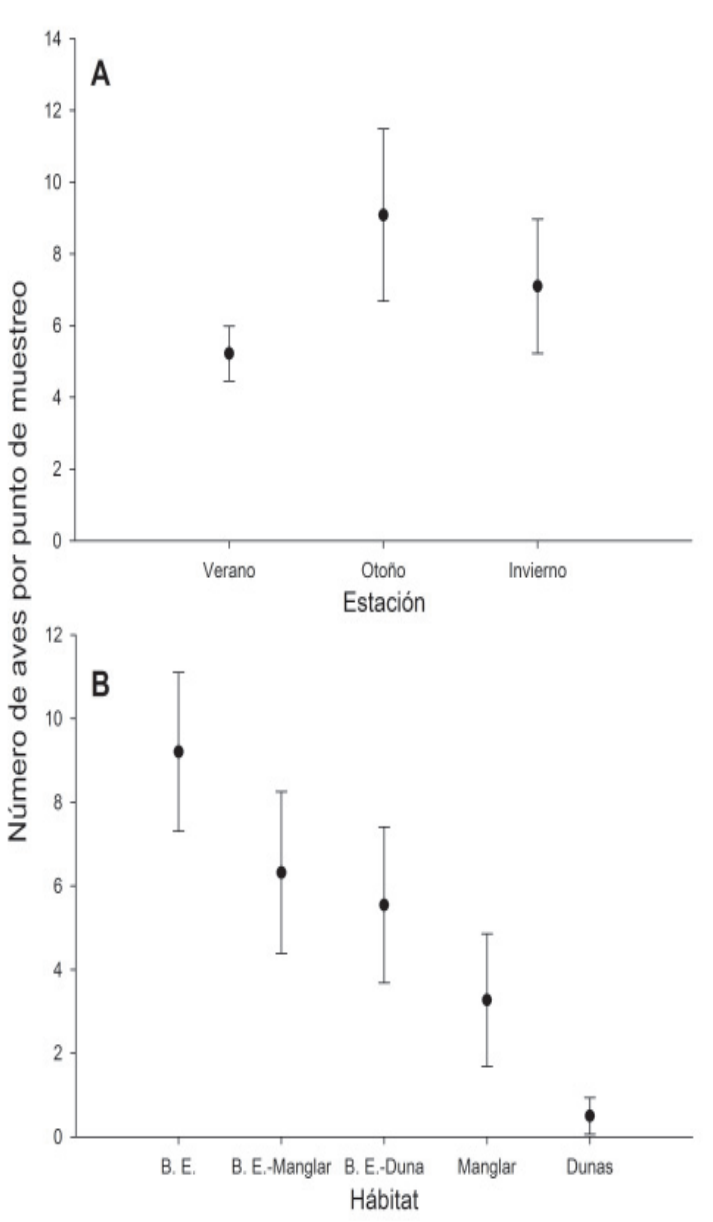

Figura 2. Abundancia promedio \pm intervalo de confianza al 95\%, en $\mathbf{A}$, las diferentes estaciones y $\mathbf{B}$, hábitats en la isla Saliaca, Sinaloa.

cardinalis y Polioptila nigriceps, y 3) migratorias ausentes en el verano y presentes en el otoño e invierno, como Aimophila carpalis, Vermivora celata y Dendroica coronata (Cuadro 3).

\section{Discusión}

Lariqueza de especies dela Isla Saliaca es comparativamente mayor que la registrada para cualquier isla del golfo de California, incluso de islas con extensiones mucho mayores, como Tiburón (79 especies, Rojas-Soto et al., 2002), Ángel de la Guarda (69 especies, Cody y Velarde, 2002) y San José (62 especies, Cody y Velarde, 2002). Este resultado puede relacionarse con 2 eventos que son difíciles de separar; por una parte, se ha documentado que las islas de la parte norte son más áridas y por lo tanto con una vegetación y disponibilidad de recursos más limitados
Cuadro 2. Abundancia promedio (aves observadas por punto de conteo) \pm Error estándar, en los diferentes hábitat y periodos de muestreo en la isla Saliaca, Sinaloa

\begin{tabular}{lcccl}
\hline \multicolumn{5}{c}{ Estación } \\
Hábitat & Verano & Otoño & Invierno & Total \\
\hline Dunas & $0.5 \pm 0.5$ & $1.3 \pm 0.3$ & --- & $1 \pm 0.2$ \\
Manglar & $5.3 \pm 1.6$ & $2.8 \pm 0.9$ & $1 \pm 0$ & $3.3 \pm 0.8$ \\
Bosque & $4.7 \pm 0.4$ & $12.4 \pm$ & $8.3 \pm 1.0$ & $9.3 \pm 0.9$ \\
espinoso & & 1.8 & & \\
BE-Duna & $6.3 \pm 0.6$ & $4.3 \pm 0.9$ & $6.4 \pm 3.9$ & $5.5 \pm 0.9$ \\
BE-Manglar & $6.2 \pm 0.8$ & $7.8 \pm 2.1$ & $4.5 \pm 1.1$ & $6.6 \pm 0.9$ \\
Total & $5.2 \pm 0.3$ & $9.2 \pm 1.2$ & $7.5 \pm 0.9$ & $7.6 \pm 0.6$ \\
\hline
\end{tabular}

para las aves (Cody y Velarde, 2002), pero puede ser producto de un esfuerzo de muestreo desigual y de la falta de información ecológica básica para la mayor parte de las islas del golfo.

Al considerar las islas de la porción sur del golfo, hay información sobre algunas localizadas en el margen oeste, cercanas a la península de Baja California: isla Carmen (54, Cody y Velarde, 2002), San José (62, Cody y Velarde, 2002), Espíritu Santo (58, Carmona et al., 2005) y Cerralvo (49, Banks, 1963). Estas islas, pese a tener un área al menos 4 veces mayor a la de Saliaca (Murphy et al., 2002), tuvieron una riqueza considerablemente menor a la registrada, debido a que en el lado peninsular predomina una vegetación de desierto, mientras que en el lado este, en el norte de Sinaloa, la precipitación es mayor a los 300 mm y la vegetación se torna más densa, alta y arbustiva, es decir, hay una transición de desierto a bosque espinoso (Johnson, 1982; Cody y Velarde, 2002) que tiene como consecuencia mayor productividad general (Cody y Velarde, 2002) y mayor cantidad de recursos disponibles para las aves. Así, aunque hace falta generar información en otras islas para corroborar este patrón, la riqueza parece ser mayor en el bosque espinoso respecto al desierto y los valores máximos de riqueza ocurrirían en islas del lado del continente.

Las variaciones estacionales en riqueza y abundancia reflejan los patrones de uso de los diferentes grupos. Así, en el verano sólo se vieron aves de reproducción local, mientras que en otoño e invierno la llegada de los migrantes neotropicales incrementó la riqueza de especies y la abundancia en los puntos de conteo. A diferencia de otras islas, como Espíritu Santo (cercanas a la península de Baja California), donde el componente migratorio es reducido (14 especies y una proporción muy baja de la abundancia total; Carmona et al., 2005) en isla Saliaca las aves migratorias tuvieron una importancia mayor en la composición de especies (30 especies). Es decir, para 
Cuadro 3. Abundancia promedio (aves por punto de conteo) de las aves terrestres observadas en los diferentes periodos de muestreo en isla Saliaca, Sinaloa

\begin{tabular}{|c|c|c|c|c|c|c|c|}
\hline \multirow[b]{2}{*}{ Especie } & \multicolumn{3}{|c|}{ Estación } & \multirow[b]{2}{*}{ Total } & \multirow[b]{2}{*}{ Hábitat } & \multirow[b]{2}{*}{$P T$} & \multirow[b]{2}{*}{$C C$} \\
\hline & Verano & Oto. & Inv. & & & & \\
\hline Cathartes aura & $\mathrm{X}$ & 0.01 & 0.12 & 0.03 & $\mathrm{BE}, \mathrm{V}$ & $\mathrm{RR}$ & $\mathrm{LC}$ \\
\hline Coragyps atratus & & & 0.02 & 0.01 & BE, V & RR & $\mathrm{LC}$ \\
\hline Pandion haliaetus & $\mathrm{X}$ & $\mathrm{X}$ & 0.12 & 0.03 & $\mathrm{BE}, \mathrm{V}$ & $\mathrm{R}$ & LC \\
\hline Circus cyaneus & & $\mathrm{X}$ & & $\mathrm{X}$ & V & M & $\mathrm{LC}$ \\
\hline Accipiter cooperii & & $\mathrm{X}$ & & $\mathrm{X}$ & $\mathrm{V}$ & M & LC Pr \\
\hline Buteo jamaicensis & & & 0.02 & 0.01 & $\mathrm{BE}, \mathrm{V}$ & M & LC \\
\hline Caracara cheriway & 0.03 & $\mathrm{X}$ & 0.05 & 0.02 & $\mathrm{BE}, \mathrm{V}$ & $\mathrm{R}$ & $\mathrm{LC}$ \\
\hline Falco sparverius & & 0.07 & 0.02 & 0.03 & $\mathrm{BE}$ & RR & $\mathrm{LC}$ \\
\hline Patagioenas flavirostris & & 0.28 & & 0.11 & $\mathrm{BE}$ & RR & $\mathrm{LC}$ \\
\hline Zenaida asiatica & 0.38 & 0.90 & 0.15 & 0.53 & $\mathrm{BE}, \mathrm{M}, \mathrm{D}$ & $\mathrm{R}$ & $\mathrm{LC}$ \\
\hline Zenaida macroura & & 0.11 & & 0.04 & $\mathrm{BE}$ & RR & $\mathrm{LC}$ \\
\hline Columbina passerina & 0.09 & 0.14 & 0.02 & 0.10 & $\mathrm{BE}, \mathrm{M}$ & $\mathrm{R}$ & $\mathrm{LC}$ \\
\hline Columbina talpacoti & $\mathrm{X}$ & 0.11 & 0.05 & 0.05 & $\mathrm{BE}$ & $\mathrm{R}$ & $\mathrm{LC}$ \\
\hline Coccyzus americanus & 0.01 & & & 0.01 & $\mathrm{BE}$ & $\mathrm{RR}$ & $\mathrm{LC}$ \\
\hline Bubo virginianus & $\mathrm{X}$ & & 0.07 & 0.02 & $\mathrm{BE}$ & RR & $\mathrm{LC}$ \\
\hline Chordeiles acutipennis & 0.05 & 0.03 & $\mathrm{X}$ & 0.03 & $\mathrm{BE}, \mathrm{M}$ & $\mathrm{R}$ & $\mathrm{LC}$ \\
\hline Cynanthus latirostris & 0.51 & 0.06 & 0.07 & 0.24 & BE, M,D & $\mathrm{RC}$ & $\mathrm{LC}$ \\
\hline Amazilia violiceps & 0.03 & & & 0.01 & $\mathrm{BE}$ & $\mathrm{RR}$ & $\mathrm{LC}$ \\
\hline Ceryle alcyon & & 0.01 & 0.05 & 0.02 & $\mathrm{BE}, \mathrm{M}$ & $\mathrm{M}$ & $\mathrm{LC}$ \\
\hline Chloroceryle americana & 0.01 & & & 0.01 & M & RR & $\mathrm{LC}$ \\
\hline Melanerpes uropygialis & & 0.03 & & 0.01 & $\mathrm{BE}, \mathrm{M}$ & $\mathrm{RR}$ & $\mathrm{LC}$ \\
\hline Picoides scalaris & 0.05 & 0.01 & & 0.03 & $\mathrm{BE}$ & RR & $\mathrm{LC}$ \\
\hline Contopus sordidulus & $\mathrm{X}$ & 0.01 & $\mathrm{X}$ & 0.01 & $\mathrm{BE}$ & $\mathrm{M}$ & $\mathrm{LC}$ \\
\hline Empidonax sp. & & 0.19 & 0.02 & 0.08 & $\mathrm{BE}$ & $\mathrm{M}$ & \\
\hline Pyrocephalus rubinus & & $\mathrm{X}$ & & $\mathrm{X}$ & BE-M & RR & $\mathrm{LC}$ \\
\hline Myiarchus tuberculifer & & & 0.02 & 0.01 & $\mathrm{BE}$ & RR & $\mathrm{LC}$ \\
\hline Myiarchus cinerascens & & 0.29 & 0.05 & 0.12 & $\mathrm{BE}$ & $\mathrm{RR}$ & $\mathrm{LC}$ \\
\hline Myiarchus tyrannulus & 0.03 & & & 0.01 & BE-D & RR & $\mathrm{LC}$ \\
\hline Pitangus sulphuratus & & 0.21 & & 0.08 & $\mathrm{BE}, \mathrm{M}$ & RR & $\mathrm{LC}$ \\
\hline Tyrannus melancholicus & 0.36 & 0.51 & & 0.34 & $\mathrm{BE}, \mathrm{M}, \mathrm{D}$ & RR & $\mathrm{LC}$ \\
\hline Tyrannus vociferans & 0.01 & & & 0.01 & $\mathrm{BE}$ & $\mathrm{M}$ & $\mathrm{LC}$ \\
\hline Tyrannus crassirostris & 0.01 & & & 0.01 & $\mathrm{BE}$ & RR & $\mathrm{LC}$ \\
\hline Lanius ludovicianus & & $\mathrm{X}$ & & $\mathrm{X}$ & $\mathrm{BE}$ & RR & $\mathrm{LC}$ \\
\hline Vireo bellii & 0.08 & 0.08 & 0.20 & 0.11 & $\mathrm{BE}, \mathrm{M}$ & M & NT A \\
\hline Corvus corax & $\mathrm{X}$ & $\mathrm{X}$ & $\mathrm{X}$ & $\mathrm{X}$ & $\mathrm{V}$ & $\mathrm{R}$ & LC \\
\hline Progne subis & $\mathrm{X}$ & & & $\mathrm{X}$ & V & M & $\mathrm{LC}$ \\
\hline Tachycineta bicolor & & & 0.37 & 0.08 & $\mathrm{BE}, \mathrm{M}$ & M & $\mathrm{LC}$ \\
\hline Tachycineta albilinea & $X$ & $\mathrm{X}$ & 0.17 & 0.04 & BE,M & $\mathrm{R}$ & LC \\
\hline
\end{tabular}

$X=$ presencia fuera de los puntos de conteo.

Hábitats donde fue observada cada especie: $\mathrm{BE}$, bosque espinoso; $\mathrm{M}$, manglar; $\mathrm{D}$, duna; $\mathrm{V}$, en vuelo.

Presencia temporal en la isla (PT): R, residente que permanece a lo largo del año en la isla; RC, residente con congregación reproductora en la isla y dispersión post-reproductiva; M, migratorio, observable sólo durante otoño e invierno y RR, residente a escala regional según Howell y Webb, 1995.

Categoría de conservación de cada especie (CC), según la IUCN (2007) y la NOM-059-ECOL-2001 (DOF, 2002) LC, least concern; NT, near threatened; Pr, protección especial y A, amenazada. 
Cuadro 3. Continúa

\begin{tabular}{|c|c|c|c|c|c|c|c|}
\hline \multirow[b]{2}{*}{ Especie } & \multicolumn{3}{|c|}{ Estación } & \multirow[b]{2}{*}{ Total } & \multirow[b]{2}{*}{ Hábitat } & \multirow[b]{2}{*}{$P T$} & \multirow[b]{2}{*}{$C C$} \\
\hline & Verano & Otoño & Invierno & & & & \\
\hline Hirundo rustica & & $\mathrm{X}$ & & $\mathrm{X}$ & $\mathrm{V}$ & M & $\mathrm{LC}$ \\
\hline Petrochelidon pyrrhonota & & $\mathrm{X}$ & & $\mathrm{X}$ & $\mathrm{V}$ & RR & $\mathrm{LC}$ \\
\hline Auriparus flaviceps & 1.25 & 0.50 & 1.39 & 0.99 & $\mathrm{M}$ & $\mathrm{R}$ & $\mathrm{LC}$ \\
\hline Campylorhynchus brunneicapillus & 0.09 & 0.10 & 0.05 & 0.08 & $\mathrm{BE}$ & $\mathrm{R}$ & $\mathrm{LC}$ \\
\hline Thryothorus sinaloa & & 0.01 & & 0.01 & $\mathrm{BE}, \mathrm{M}$ & $\mathrm{RR}$ & $\mathrm{LC}$ \\
\hline Thryothorus felix & & 0.06 & 0.05 & 0.03 & BE, M & $\mathrm{RR}$ & $\mathrm{LC}$ \\
\hline Troglodytes aedon & & & 0.02 & 0.01 & $\mathrm{BE}$ & M & $\mathrm{LC}$ \\
\hline Polioptila caerulea & & & 0.22 & 0.05 & $\mathrm{BE}$ & M & $\mathrm{LC}$ \\
\hline Polioptila nigriceps & 0.61 & 0.63 & 0.71 & 0.63 & BE,M & $\mathrm{R}$ & $\mathrm{LC}$ \\
\hline Turdus rufopalliatus & & 0.18 & 0.02 & 0.07 & $\mathrm{BE}$ & RR & $\mathrm{LC}$ \\
\hline Mimus polyglottos & 0.13 & 0.56 & 0.54 & 0.38 & $\mathrm{BE}, \mathrm{M}$ & $\mathrm{R}$ & $\mathrm{LC}$ \\
\hline Toxostoma curvirostre & & 0.04 & 0.02 & 0.02 & $\mathrm{BE}$ & $\mathrm{RR}$ & $\mathrm{LC}$ \\
\hline Vermivora celata & & 0.71 & 0.29 & 0.33 & $\mathrm{BE}, \mathrm{M}$ & $\mathrm{M}$ & $\mathrm{LC}$ \\
\hline Dendroica petechia & & 0.22 & 0.02 & 0.09 & $\mathrm{BE}$ & M & $\mathrm{LC}$ \\
\hline Dendroica p. erithachorides & 0.03 & $\mathrm{X}$ & $\mathrm{X}$ & 0.01 & $\mathrm{M}$ & $\mathrm{R}$ & \\
\hline Dendroica coronata & & $\mathrm{X}$ & $\mathrm{X}$ & $\mathrm{X}$ & $\mathrm{BE}$ & M & $\mathrm{LC}$ \\
\hline Mniotilta varia & & 0.04 & & 0.02 & $\mathrm{BE}, \mathrm{M}$ & M & \\
\hline Seiurus noveboracensis & & $\mathrm{X}$ & & $\mathrm{X}$ & $\mathrm{BE}, \mathrm{M}$ & M & $\mathrm{LC}$ \\
\hline Oporornis tolmiei & & 0.03 & & 0.01 & $\mathrm{BE}$ & M & LC A \\
\hline Geothlypis trichas & 0.01 & 0.04 & 0.05 & 0.03 & $\mathrm{BE}, \mathrm{M}$ & $\mathrm{R}$ & LC \\
\hline Wilsonia pusilla & & 0.04 & 0.07 & 0.03 & $\mathrm{BE}$ & M & $\mathrm{LC}$ \\
\hline Piranga rubra & & & $\mathrm{X}$ & $\mathrm{X}$ & $\mathrm{BE}$ & M & $\mathrm{LC}$ \\
\hline Pipilo chlorurus & & 0.08 & 0.10 & 0.05 & $\mathrm{BE}$ & M & $\mathrm{LC}$ \\
\hline Aimophila carpalis & 0.13 & 0.51 & 0.05 & 0.26 & $\mathrm{BE}, \mathrm{M}$ & $\mathrm{RC}$ & $\mathrm{LC}$ \\
\hline Aimophila botterii & & $\mathrm{X}$ & 0.02 & 0.01 & $\mathrm{BE}$ & $\mathrm{M}$ & $\mathrm{LC}$ \\
\hline Aimophila ruficeps & & 0.06 & 0.05 & 0.03 & $\mathrm{BE}$ & M & $\mathrm{LC}$ \\
\hline Spizella passerina & & $\mathrm{X}$ & 0.41 & 0.09 & $\mathrm{BE}$ & M & $\mathrm{LC}$ \\
\hline Spizella pallida & & & $\mathrm{X}$ & $\mathrm{X}$ & $\mathrm{BE}$ & M & $\mathrm{LC}$ \\
\hline Chondestes grammacus & & & 0.02 & 0.01 & $\mathrm{BE}$ & M & $\mathrm{LC}$ \\
\hline Passerculus sandwichensis & & $\mathrm{X}$ & & $\mathrm{X}$ & $\mathrm{M}$ & $\mathrm{M}$ & $\mathrm{LC}$ \\
\hline Saltator coerulescens & & 0.03 & & 0.01 & $\mathrm{BE}$ & $\mathrm{RR}$ & $\mathrm{LC}$ \\
\hline Cardinalis cardinalis & 0.91 & 0.76 & 0.54 & 0.77 & $\mathrm{BE}, \mathrm{M}$ & $\mathrm{RC}$ & $\mathrm{LC}$ \\
\hline Pheucticus melanocephalus & & 0.03 & & 0.01 & $\mathrm{BE}$ & RR & $\mathrm{LC}$ \\
\hline Passerina versicolor & 0.54 & 0.26 & 0.10 & 0.34 & $\mathrm{BE}$ & $\mathrm{RC}$ & $\mathrm{LC}$ \\
\hline Passerina ciris & & 0.14 & 0.02 & 0.06 & $\mathrm{BE}$ & $\mathrm{M}$ & NT \\
\hline Quiscalus mexicanus & 0.24 & & 0.05 & 0.11 & $\mathrm{BE}$ & RR & $\mathrm{LC}$ \\
\hline Molothrus aeneus & 0.11 & & & 0.04 & $\mathrm{BE}, \mathrm{M}$ & $\mathrm{RR}$ & $\mathrm{LC}$ \\
\hline Molothrus ater & $\mathrm{X}$ & 0.13 & 0.32 & 0.12 & $\mathrm{BE}, \mathrm{M}$ & $\mathrm{R}$ & $\mathrm{LC}$ \\
\hline Icterus cucullatus & 0.05 & 0.01 & & 0.03 & $\mathrm{BE}, \mathrm{M}$ & RR & $\mathrm{LC}$ \\
\hline Icterus pustulatus & 0.04 & 0.29 & 0.10 & 0.15 & $\mathrm{BE}$ & $\mathrm{R}$ & $\mathrm{LC}$ \\
\hline Icterus bullockii & & 0.01 & & 0.01 & $\mathrm{BE}, \mathrm{M}$ & M & $\mathrm{LC}$ \\
\hline Carpodacus mexicanus & 0.04 & 0.47 & 0.27 & 0.25 & $\mathrm{BE}, \mathrm{M}$ & $\mathrm{R}$ & $\mathrm{LC}$ \\
\hline Carduelis psaltria & & 0.24 & 0.02 & 0.10 & $\mathrm{BE}$ & $\mathrm{RR}$ & $\mathrm{LC}$ \\
\hline Abundancia promedio & 5.83 & 9.25 & 7.32 & 7.46 & & & \\
\hline Riqueza & 33 & 59 & 48 & 82 & & & \\
\hline
\end{tabular}


las aves migratorias, las islas del margen este del golfo parecen ser más importantes que las cercanas a la península de Baja California.

Nuestros resultados muestran que el bosque espinoso es el hábitat más usado por las aves. Recurrentemente se ha demostrado que tanto el área como la complejidad o heterogeneidad del hábitat se correlacionan positivamente con la riqueza y abundancia de especies (Mac Nally y Watson, 1997; Rahbek y Graves, 2001), lo que explicaría las diferencias observadas en Saliaca, pues el bosque espinoso es el hábitat con mayor complejidad y cobertura en la isla (véase Área de estudio), seguido por los ecotonos de éste con los otros hábitat y finalmente el mangle y la duna, es decir, el ordenamiento repetido en los análisis de riqueza y abundancia.

En cuanto a los patrones observados para las diferentes especies; para aquellas con abundancia mayor en el verano, como Auriparus flaviceps, Cynanthus latirostris y Passerina versicolor, la isla parece ser una zona de congregación reproductiva, y la disminución de su abundancia en el otoño e invierno indicaría una dispersión postreproductiva, muy probablemente hacia la zona costera del continente donde estas especies son comunes a lo largo del año (M. Guevara, datos no publicados). Los migrantes neotropicales como chipes y gorriones usan la zona para pasar el invierno después de reproducirse en zonas norteñas, así que formaron un componente importante sólo durante otoño e invierno. Finalmente, hay especies que se mantienen en la zona con abundancias similares todo el año, como Cardinalis cardinalis y Polioptila nigriceps, en ese sentido son más dependientes de la isla, y las que resentirían más severamente la pérdida de hábitat.

En general, la composición de especies en la isla parece similar a lo que se puede observar en la zona costera y sólo Accipiter coperii, Vireo bellii y Oporornis tolmiei están consideradas como especies con algún riesgo de conservación (IUCN, 2007, DOF, 2002; Cuadro 3). Pese a lo anterior, la isla Saliaca, al menos a escala regional, parece ser un segmento con alto valor para la conservación pues su vegetación corresponde con la de las tierras continentales cercanas (Cody et al., 2002), mismas que han sido fuertemente modificadas para uso agrícola y acuícola en la región (Conservación Internacional, 2003). Así, los hábitat de la isla y su composición de especies representarían un relicto de los hábitats de vegetación costera sinaloense.

\section{Agradecimientos}

A la CONANP y CONACYT, por el apoyo financiero. A la consultora Oikos (en particular Edna Carrillo) por gestionar los recursos necesarios y por el apoyo logístico brindado. A Aarón Aguilar, Fabiola Cárdenas, Roxana D. Espinoza, Josúe Robles, Gabriel López, Anabel Buelna, Jesús Sandoval, Miguel A. Guevara y Sarahí López, por su invaluable ayuda en el trabajo de campo. A José Alfredo Navarrete, por su ayuda en la identificación de plantas y caracterización del hábitat.

\section{Literatura citada}

AOU (American Ornithologists' Union). 1998. Check-list of North American birds. Allen Lawrence, Kansas.

Anderson, D. W. 1983. The seabirds. In Island biogeography in the Sea of Cortez, T. J. Case y M. L. Cody (eds.). University of California Press, Berkeley. p. 46-264.

Banks, R. C. 1963. Birds of Cerralvo Island, Baja California. The Condor 65:300-312.

Brewster, W. 1902. Birds of the Cape region of lower California. Bulletin of the Museum of Comparative Zoology 41:1-241

Carmona, R., G. Ruiz-Campos, J. A. Castillo-Guerrero y G. Brabata. 2005. patterns of occurrence and abundance of land birds on Espiritu Santo Island, Gulf of California, México. The Southwestern Naturalist 50:440-447.

Casagrande, D. G., y S. R. Beissinger. 1997. Evaluation of four methods for estimating parrot population size. The Condor 99:445-457.

Cody, M. L., y E. Velarde. 2002. Land birds. In A new island biogeography of the Sea of Cortés. T. J. Case, M. L. Cody y E. Ezcurra (eds.) Oxford University Press, New York. p. $271-312$.

Colwell, R. K. 2006. EstimateS, Version 7: Statistical estimation of species richness and shared species from samples (software and user's guide). Freeware for Windows and Mac OS.

Conservación Internacional. 2003. Conservación y desarrollo para bahía Santa María, Sinaloa. Estrategia de manejo. Comisión Conservación y Desarrollo Bahía Santa María, México. Guaymas, Sonora.

De la Lanza-Espino, G. y C. Cáceres-Martínez. 1994. Lagunas costeras y el litoral mexicano. Universidad Autónoma de Baja California Sur, La Paz. 525 p.

DOF. 1978. Decreto por el que se establece una zona de reserva y refugio de aves migratorias y de la fauna silvestre, en las islas que se relacionan, situadas en el golfo de California. Diario Oficial de la Federación, 2 de agosto de 1978, México, D. F.

DOF. 2002. Norma Oficial Mexicana NOM-059-ECOL-2001, Protección ambiental. Especies nativas de México de flora y fauna silvestres. Categorías de riesgo y especificaciones para su inclusión, exclusión o cambio. Lista de especies en riesgo, Diario Oficial de la Federación, 6 de marzo de 2002, México, D. F.

Gaviño, G., F. Aguilar, y L. C. García. 1984. Abundancia relativa, reproducción y actividad diurna de aves terrestres en la Isla Carmen, Baja California Sur, México. Anales del Instituto de Biología, Universidad Nacional Autónoma de México, Serie Zoología 55:263-284. 
Grinnell, J. 1928. A distributional summation of the ornithology of lower California. University of California. Publications in Zoology 32:1-300.

Howell, S. N. G. y S. Webb.1995. A guide to the birds of Mexico and Northern Central America. Oxford Unniversity Press, New York. 851 p.

IUCN. 2007. IUCN Red list of threatened species. www/ iucnredlist.org/ consultada 18 de enero, 2008.

Jiménez-Valverde, A. y J. Hortal. 2003. Las curvas de acumulación de especies y la necesidad de evaluar la calidad de los inventarios biológicos. Revista Ibérica de Aracnología 8:151-161.

Johnson, A. F. 1982. Dune vegetation along the eastern shore of the Gulf of California. Journal of Biogeography 9:317-330.

Mac Nally, R. y D. M. Watson. 1997. Distinguishing area and habitat heterogeneity effects on species richness: birds in Victorian buloke remnants. Austral Journal of Ecology 22:227-232.

Massey, B. W. y E. Palacios. 1994. Avifauna of the wetlands of Baja California, Mexico: current status. Studies in Avian Biology 15:45-57.

Mikol, S. A. 1980. Field guidelines for using transects to sample nongame bird populations. FWS/OBS-80/58, United States Department of the Interior, Fish and Wildlife Service, Biological Services Program, Washington, D.C.

Murphy, R. W., F. Sanchez-Piñero, G. A. Polis y R. L. Aalbu. 2002. New measurements of Area and distance for islands in the Sea of Cortés. In A new island biogeography of the Sea of Cortés. T. J. Case, M. L. Cody y E. Ezcurra (eds.) Oxford University Press, New York. p. 271-312.

Rahbek, C. y G. R. Graves. 2001. Multiscale assessment of patterns of avian species richness. Proceedings of the National Academy of Sciences 98: 4534-4539.
Ralph, C. J., G. R. Geupel, P. Pyle, T. E. Martin, D. F. DeSante y B. Mila. 1996. Manual de métodos de campo para el monitoreo de aves terrestres. General technical report. SWGTR-159, U. S. Department of Agriculture, Forest Service, Pacific Southwest Research Station, Albany, California.

Rojas-Soto, O. R., F. Puebla-Olivares, E. M. Figueroa-Esquivel, L. A. Sánchez-González, Y. J. Nakazawa-Ueji, C. A. RíosMuñoz y A. G. Navarro-Singüenza. 2002. Avifauna de Isla Tiburón, Sonora, México. Anales del Instituto de Biología, Universidad Nacional Autónoma de México. Serie Zoología 73:73-89.

Rzedowski, J. 1978. Vegetación de México. Limusa, México, D. F. 423 p.

Serrano D. y E. Ramírez-Félix. 2003. Implementación de un modelo hidrodinámico en el sistema lagunar de Santa María La Reforma, Sinaloa. Inpesca 1:33-39.

Shiu, H. J. y P. F. Lee. 2003. Assessing avian point-count duration and sample size using species accumulation functions. Zoological Studies 42:357-367.

Sibley, D. A. 2001. The Sibley guide to birds. Knopf, New York.

StatSoft, 2005. Statistica (data analysis software system), version 7.1. www.statsoft.com.

Tershy, B. R., D. Breese y D. Croll. 1997. Human perturbations and conservation strategies for San Pedro Mártir Island, Islas del Golfo de California Reserve, México. Envinronmental Conservation 24 :261-270.

Velarde, E. y D. W. Anderson. 1994. Conservation and management of seabird islands in the Gulf of California: setbacks and successes. In Seabirds on islands: treats, case studies and action plans. D. N. Nettleship, J. Burger y M. Gochfeld (eds.). Technical publication. ICPB, Cambridge. p. 229-243. 